\title{
Aprendizaje Servicio en la Formación Inicial de Docentes en la Universidad de Huelva. El Proyecto INCLUREC
}

\section{Service Learning in Special Education Teachers' Initial Training at the University of Huelva. INCLUREC Project}

\author{
Inmaculada Gómez-Hurtado * \\ Asunción Moya Maya \\ M. Pilar García-Rodríguez \\ Universidad de Huelva, España
}

\begin{abstract}
La presente investigación describe la experiencia didáctica de aprendizaje servicio desarrollada en la Universidad de Huelva ligada al Proyecto de Innovación Docente INCLUREC en la que participan estudiantes de universidad, alumnado con necesidades educativas especiales y profesorado de diferentes niveles educativos. La experiencia parte de la elaboración y adaptación de recursos didácticos para el alumnado con necesidades educativas, tratando de desarrollar una formación universitaria comprometida, funcional y de servicio a estas personas y a la sociedad, y creando una red de colaboración entre universidad, escuelas y asociaciones desde una perspectiva de accesibilidad universal. La experiencia se evalúa mediante el análisis de más de 600 fichas elaboradas por el alumnado, notas académicas y cuatro entrevistas a informantes clave. Los resultados abordan las necesidades del alumnado, de recursos, de los centros que han colaborado y acerca de la formación del alumnado universitario participante. Se concluye destacando los buenos resultados académicos del alumnado universitario, la conexión con profesionales a los que la experiencia les sirve para su formación permanente y la importante contribución de recursos materiales para los estudiantes con necesidades educativas especiales.
\end{abstract}

Descriptores: Enseñanza Superior; Relación escuela-comunidad; Necesidades Educacionales; Recursos educativos.

This research describes the didactic learning experience developed in the University of Huelva linked to the Teaching Innovation Project INCLUREC in which have participated university students, students with special educational needs and a teacher of different educational levels. The experience starts from the elaboration and adaptation of didactic resources to the student with educational needs, trying to develop a compromised, functional and functional university formation and to create a collaborative network between the university, the students and the associations since a perspective of universal accessibility. The experience evolves through the analysis of more than 600 records prepared by a student, academic rates and four interviews with key informants. The results address the needs of the students, resources, schools that have collaborated and about the formation of participating university students. It concludes by highlighting the good academic rates of the university student, the connection with professionals to those who this experience serve them as permanent formation and the important contribution of material resources to students with special educational needs.

Keywords: Higher education; School community relationship; Special needs; Educational resources.

*Contacto: inmaculada.gomez@ddcc.uhu.es 


\section{Introducción}

La enseñanza universitaria ha estado sometida a múltiples cambios en las dos últimas décadas: catálogo de títulos, planes de estudio, nuevos modelos de programaciones, metodologías docentes, enseñanza centrada en el alumnado reestructuración horaria, conocimiento de idiomas... Con estos cambios, uno de los objetivos estratégicos que se ha perseguido ha sido la "mejora de la calidad y la eficacia de la educación y la formación", siendo algunas de las acciones necesarias, las de garantizar una docencia de alta calidad, una formación inicial adecuada y un desarrollo profesional continuo de profesores y formadores y hacer de la docencia una opción atractiva de carrera (Consejo de la Unión Europea, 2009). Desde el marco europeo se insta a las instituciones universitarias a que promuevan cambios en su docencia (Comisión de las Comunidades Europeas, 2005).

Son muchos los estudios que se vienen desarrollando para mejorar los rendimientos de los estudiantes universitarios y ello implica revisar los modelos de aprendizaje y las metodologías de enseñanza entre otros factores (Gargallo López et al., 2017; Monroy y Hernández Pina, 2014). Es así como surge nuestro proyecto de introducción del enfoque de aprendizaje servicio (ApS) en la Facultad de Ciencias de la Educación de la Universidad de Huelva. En un primer momento para la formación inicial de futuros maestros y maestras de la mención de Educación Especial, para más tarde llevarlo también a otras especialidades y a la formación del alumnado del Máster de Educación Especial. Quince han sido las materias implicadas en estos años de desarrollo de INCLUREC (recursos para la inclusión) y con diferentes grados de implicación.

El proyecto forma parte del cambio de paradigma docente que estamos viviendo en el ámbito universitario: pasamos de un paradigma centrado en la instrucción a otro focalizado en el aprendizaje (Gargallo López et al., 2017). De acuerdo con Murillo (2012), consideramos que, para conseguir una enseñanza universitaria adecuada en la formación de maestros y maestras, para construir una escuela de calidad, debemos abogar por el desarrollo de principios inclusivos, por la eficacia y la innovación. Esto implica la construcción de una escuela para todos y todas, donde se mejoren los procesos de enseñanza y aprendizaje y se dé la justicia social. La colaboración es uno de los principios de este proyecto que presentamos y es una de las bases de la inclusión escolar (Murillo y Hernández-Castilla, 2014).

La motivación inicial del proyecto era nuestra preocupación por la reducción de créditos formativos relacionados con contenidos específicos de la especialidad de Educación Especial que se había producido con el cambio de planes de estudios. El antiguo Plan de Estudios de Maestros de Educación Especial contaba con 240 créditos en su mayoría orientados a la formación para la atención de personas con necesidades especiales. El nuevo plan de estudios ofrecía una formación generalista en Educación Primaria, con sólo 30 créditos de formación específica inscritos en la mención de Educación Especial. Concretamente se ofrecen cinco materias con seis créditos cada una; éstas tienen carácter optativo, pudiendo ser elegidas por el alumnado desde segundo a cuarto curso para obtener la citada mención.

Esta nueva estructura y contenidos han implicado cambios en el desarrollo de las materias para alcanzar las competencias propuestas, así como para responder a las exigencias del crédito europeo. Estas transformaciones, así como el desarrollo de experiencias de colaboración con los centros educativos y con los Equipos de Orientación Educativa 
(EOES), van conformando y definiendo una metodología basada en la colaboración universidad-centros y una formación orientada hacia la respuesta educativa concreta y real. Estos planteamientos nos han llevado hacia un enfoque y un proceso de aprendizaje y enseñanza que, en muchos aspectos, coincide con los de aprendizaje servicio. La metodología de trabajo es semejante a la descrita por Puig (2009); partimos de la detección de necesidades que se demandan (alumno/a con discapacidad para el que se le solicita la creación/adaptación de un recurso educativo), la planificación de las respuestas a las mismas y la adquisición de contenidos, competencias y valores propios de la titulación que cursa el estudiante.

Para la presentación de esta experiencia y sus resultados iniciales hemos seguido las recomendaciones de Murillo, Martínez-Garrido y Belavi (2017). A lo largo de este trabajo describimos cómo introducimos el Aprendizaje servicio (ApS) en nuestros títulos y destacamos los resultados más significativos hallados en el proceso de evaluación que estamos llevando a cabo.

\title{
1. ApS aplicado a la formación inicial de maestros en la UHU
}

Coincidimos con Puig y otros (2009) en que el ApS es:

\begin{abstract}
Una metodología pedagógica de alto poder formativo. Una metodología que combina en una sola actividad el aprendizaje de contenidos, competencias y valores con la realización de tareas de servicio a la comunidad. En el aprendizaje servicio el conocimiento se utiliza para mejorar algo de la comunidad y el servicio se convierte en una experiencia de aprendizaje que proporciona conocimientos y valores. Aprendizaje y servicio quedan vinculados por una relación circular en la que ambas partes salen beneficiadas: el aprendizaje adquiere sentido cívico y el servicio se convierte en un taller de valores y saberes. (p. 9)
\end{abstract}

De esta forma podemos decir, que constituye un proyecto y acuerdo colaborativo con la comunidad buscando siempre alcanzar los objetivos comunes de la misma para dar respuesta a una necesidad real no cubierta (Puig et al., 2011).

Internacionalmente, el ApS se define por tres rasgos sobre los que la reflexión se hace fundamental (Mayor Paredes y Rodríguez Mar, 2015; Montes, Tapia y Yaber, 2011 ; Páez y Puig, 2013; Puig, 2009):

- Se trata de un servicio a la comunidad con el propósito de dar respuesta a necesidades reales y sentidas.

- Protagonismo de los y las participantes (estudiantado de distintos niveles educativos, docentes, representantes de entidades sociales y recursos públicos), en la detección de necesidades, planificación de respuestas a éstas, así como en la adquisición de contenidos, competencias y valores propios de la titulación que cursan los estudiantes.

- Planificación intencional e integrada de los contenidos curriculares y las actividades que conforman el servicio a la comunidad.

Para garantizar el éxito de estas prácticas, es necesario lograr un equilibrio entre los aprendizajes del estudiante y el servicio orientado a responder una necesidad real de una comunidad (Páez y Puig, 2013); ambas partes deben beneficiarse y retroalimentarse. 
El aprendizaje colaborativo según Kugelmass (2001), es necesario para que los estudiantes puedan unir los conocimientos e ideas con situaciones de colaboración, resolución de problemas y creación de nuevo conocimiento. Para ello, se necesitan experiencias en Educación Superior que promuevan estos aspectos (Häkkinen et al., 2017). Paralelamente, la participación en estas experiencias de ApS incrementa el interés en temáticas como la diversidad, reduciendo estereotipos y aumentando la sensibilidad hacia problemas tales como la adaptación del currículum -según las demandas de los jóvenes- (Root, 2005)

García y Cotrina (2015) defienden este enfoque de trabajo en el campo de la Educación. Parten de la consideración de que la educación, en su dimensión social y cívica, ha de ser construida de forma activa. Para nuestro equipo de trabajo, el ApS es una herramienta fundamental en el desarrollo de las competencias docentes y en la construcción de la ética profesional y la responsabilidad social con el entorno que tiene la propia universidad como institución. En este sentido, autores como Rodríguez Herrero, de la Herrán y Cortina Selva (2015, p. 195), hablan de “...metodología pedagógica activa que integra actividades de formación complejas para la evolución personal y de la humanidad, desarrolladas tanto en el contexto escolar como en el servicio a otros entornos de la comunidad". Y Gil-Gómez y otros (2016) enfatizan en el ApS su contribución sobre la formación ciudadana en busca de un bien común.

Unificando este marco conceptual, concebimos el ApS siguiendo la descripción de Aramburuzabala (2013), como herramienta de aprendizaje y de transformación social, ya que posibilita que estudiantes y docentes aprendan en contacto con la realidad mientras que atienden las necesidades del entorno. Para darles respuesta, realizan cambios importantes mediante acciones de mejora, a la vez que reflexionan con una guía por parte del profesor o profesora de la experiencia vivida. En nuestro proyecto, además, la aplicación de este enfoque ha significado dar respuestas eficaces a los Principios europeos comunes para las competencias y cualificaciones del profesorado (Unión Europea, 2007).

El proyecto INCLUREC, trata por un lado, de proporcionar al alumnado universitario participante capacidades y competencias: aplicación de conocimientos a su trabajo o vocación de una forma profesional; desarrollar competencias que suelen demostrarse por medio de la elaboración y defensa de argumentos; resolución de problemas dentro de su área de estudio; y aprender a aprender resolviendo problemas de forma efectiva, trabajando de forma autónoma y colaborativa, organizando, planificando y tomando decisiones. Por otro, trata de dar respuesta a las necesidades concretas de determinados alumnos y alumnas en relación con sus limitaciones y necesidades educativas, como más adelante podremos comprobar. De ahí que trabajemos sobre:

- Aprendizajes guiados: el estudiante debe investigar y completar su formación en función del caso sobre el que esté trabajando. Ello implica la adquisición de estrategias para el aprendizaje a lo largo de la vida.

- Aplicación del aprendizaje por competencias.

- Trabajo cooperativo del alumnado tanto en el taller, como con los centros educativos. Ello supone vincular la formación inicial con la permanente del profesorado en ejercicio, y la creación de redes de colaboración entre centros.

- Elaboración y seguimiento de un recurso para un alumno/a con discapacidad, que implica un trabajo "en", "para" y "con" la sociedad. 
- La mejora de la calidad de la formación de nuestro alumnado, adquiriendo estrategias de aprender a aprender, ya que deben dar respuestas reales a una sociedad cambiante de manera autónoma.

\section{Desarrollo de la experiencia: El proyecto INCLUREC}

El proyecto nace en el curso 2007-2008 llegando hasta la actualidad y afianzándose como experiencia de aprendizaje servicio. Se constituye como una propuesta educativa en la que los participantes se forman y trabajan sobre necesidades reales del entorno con el fin de mejorarlo (Puig y Palos, 2006). Se inició evaluando y creando recursos para alumnado con limitaciones de la movilidad (hasta el curso 2014-2015), para más tarde (desde 2015 y hasta hoy) diversificar los destinatarios con cualquier necesidad específica de apoyo educativo en relación a recursos educativos.

Este proyecto es financiado y avalado por la propia universidad, concretamente por la convocatoria pública competitiva del Plan Propio de la misma de cada año académico. También ha contado en ocasiones con aportaciones de otras entidades públicas y privadas. Además, conlleva el establecimiento de convenios con diferentes entidades tales como la Consejería de Educación o delegación de la provincia, concretamente los equipos de orientación educativa y departamentos de orientación de los centros de Educación Infantil, Primaria y Secundaria, los Centros de Formación del Profesorado y, por otra parte, diferentes asociaciones y entidades sin ánimo de lucro.

\subsection{Objetivos de la experiencia}

Tanto los recursos personales como los materiales forman parte de los elementos de acceso al currículo; son imprescindibles para el desarrollo del proceso educativo y deben servir para responder a las necesidades educativas de todo el alumnado. Es por ello necesario rentabilizar al máximo los recursos ordinarios, que son aquellos que existen de manera habitual en los centros (ya que muchos de los alumnos y alumnas con necesidades no precisarán de recursos específicos), y crear o adaptar otros, para aquellos alumnos/as con necesidades educativas especiales atendiendo a sus peculiaridades, limitaciones y posibilidades.

De aquí surge el objetivo principal de este proyecto: estudiamos y analizamos las necesidades de diferentes personas con necesidades educativas especiales para darles respuesta, a través de la adaptación de recursos. Para el desarrollo de esta meta, concretamos como objetivos a conseguir:

- Concienciar al alumnado universitario sobre la necesidad de un "diseño universal" respecto a los juegos y juguetes que se desarrollarán en Infantil, Primaria y Secundaria, donde todos los alumnos y alumnas puedan participar independientemente de sus posibilidades y limitaciones.

- Eliminar las barreras para el alumnado con necesidades educativas a la hora de participar en juegos tanto en su aula como en el patio de recreo.

- Preparar, seleccionar o construir materiales didácticos y de comunicación para la inclusión escolar y social.

- Desarrollar la capacidad para actuar en situaciones reales ante las necesidades de alumnos alumnas con necesidades de apoyo educativo. 
- Poner al alumnado de la Facultad en contacto con materiales ya elaborados y adaptados a este alumnado.

- Favorecer la coordinación y colaboración entre los centros educativos, asociaciones u organizaciones sin ánimo de lucro que atienden la diversidad del alumnado y la Universidad.

- Formar y sensibilizar al alumnado en general de la Universidad respecto a las personas con discapacidad.

\subsection{Metodología implementada en la experiencia}

Como adelantábamos, la realidad de la universidad en el Espacio Europeo de Educación Superior demanda nuevas metodologías didácticas a implementar en las aulas. De ahí que recurramos a metodologías activas como el ApS.

La docencia en la Universidad necesita este cambio de metodologías de enseñanzaaprendizaje, que permitan el desarrollo de los contenidos y competencias, y el aprendizaje de los mismos por parte del alumnado. Este tipo de metodologías que centra la enseñanza en los aprendizajes, en lograr el aprendizaje autónomo del alumnado partiendo de sus intereses, mirando hacia los resultados -entendiendo éstos como adquisición de competencias generales y especificas-, trata de promocionar el trabajo cooperativo, definiendo actividades de enseñanza-aprendizaje; conceptualiza la evaluación como mejora en el proceso y otorga gran importancia a las tecnologías de la información y comunicación en el proceso (Fernández March, 2006). Esto supone la implicación en experiencias innovadoras que den respuesta a las nuevas necesidades del alumnado (Bigg, 2003; Ponsa et al., 2009; Rué, 2006, Sánchez Moreno, 2007; Ubieto et al., 2008; Zabalza y Zabalza, 2010).

Los principios del ApS de los que partimos se focalizan en considerar prioritaria la detección de las necesidades que se demandan, la planificación de las respuestas a las mismas y la adquisición de contenidos, competencias y valores propios de la titulación que cursa el estudiante (Puig, 2009). Partiendo de esto, nos basamos en:

- El fomento de la experiencia y reflexión.

- Servicio real a la comunidad.

- La metodología activa y participativa.

- Trabajo en grupo.

- Trabajos prácticos como base de la formación teórica adquirida.

- Partir de las necesidades de alumnos y alumnas concretas de los centros de Primaria y Secundaria de Huelva capital y provincia.

- Trabajar en entornos de aprendizaje externos (centros escolares) e internos (taller de la universidad)

- Trabajo focalizado en el desarrollo personal, social y educativo.

\subsection{Actividades realizadas desde la perspectiva de aprendizaje servicio}

Si definimos el Aprendizaje Servicio como "una actividad o programa de servicio solidario protagonizado por los estudiantes, orientado a atender eficazmente las necesidades de una comunidad, y planificada de forma integrada con los contenidos curriculares con el objetivo de optimizar los aprendizajes" (Tapia, 2008, p. 43), el proceso que seguimos en el 
proyecto INCLUREC se puede describir y concretar en diferentes actividades. El proceso comienza con la formación y organización de los grupos de alumnado de las asignaturas implicadas. Les informamos de los objetivos del proyecto, su relación con las asignaturas, criterios de evaluación... Es esencial la formación en algunas de las competencias que les van a ser necesarias para responder a las demandas de los centros. Con este objetivo se les muestran recursos elaborados por el alumnado en otros cursos, se explican las principales demandas realizadas por los centros y se les forma de manera práctica para responder a estas posibles demandas. Esta formación estará referida a la realización de las adaptaciones más básicas de material (juguetes para la comunicación, sistemas alternativos de comunicación, adaptaciones para la manipulación, adaptaciones de material escolar, sistemas de imprentillas, etc.) Partiendo de estas acciones introductorias, la secuencia de actividades que seguimos son:

- Comenzamos con el contacto con los centros tanto de Huelva capital como de la provincia, con el objetivo de determinar las necesidades de recursos de bajo coste que estos detectan respecto al alumnado con necesidades específicas de apoyo educativo. Este contacto tiene varias modalidades: el centro se pone en contacto con el proyecto, el alumnado indica centros que podrían estar interesados y también los propios equipos de orientación indican y difunden el proyecto.

- El grupo de alumnado de la Universidad, acuerda con el profesorado del centro asignado (profesorado de apoyo, tutores...) a qué alumnos y alumnas va a dirigirse el trabajo de adaptación y elaboración de recursos, sus necesidades, la adaptación necesaria, al uso que se le dará, sus finalidades... Para esta etapa se emplea una guía de entrevista y de observación con el objetivo de delimitar y analizar las necesidades de este profesor/a y la realidad del alumno/a.

- Una vez concretados y acordados los recursos a realizar, sus objetivos, necesidades y destinatarios, definimos y organizamos el plan de trabajo en relación a la elaboración del recurso o recursos. En esta fase la formación es imprescindible para poder dar una respuesta adecuada, cumpliéndose así uno de los requisitos del Aprendizaje Servicio (Gil-Gómez et al., 2016).

- Elaboración o adaptación de los recursos concretos con la supervisión y tutorización de las profesoras usando los materiales con los que cuenta el aula taller situado en el Departamento de Pedagogía de la Universidad de Huelva. Este proceso tiene una duración variable dependiendo del recurso, la dificultad... Durante este proceso se recomienda al alumnado que mantenga un contacto personal o por medio de correo, teléfono, etc., con el centro para mostrar bocetos, aclarar dudas...

- Después de llevar el material elaborado al centro y "probarlo" con el alumnado destinatario para las modificaciones precisas y sugeridas, es presentado al resto de compañeros/as de la clase en unas Jornadas destinadas a esta finalidad. El alumnado universitario implicado describe las necesidades del alumnado destinatario, los objetivos del recurso, dificultades y sugerencias de elaboración... para responder a las limitaciones detectadas desde la perspectiva de aprendizaje servicio. Todo este proceso queda recogido también en una "ficha técnica" del material donde se recogen las características y uso con los siguientes apartados: destinatario, edad, objetivos a desarrollar, desarrollo y orientaciones didácticas. 
- El material elaborado se cede al centro donde se escolariza el alumnado destinatario de la adaptación y que pretende dar respuesta a las necesidades indicadas: estimulación sensorial, autonomía personal y social, comunicación, desarrollo instrumental...que desarrollan los principios del aprendizaje servicio. El centro rellena un cuestionario de valoración de la experiencia y del recurso/s, evaluando la adecuación, uso del material, etc., pudiendo hacer propuestas de modificación de aquellos elementos que el profesor considere necesarios.

\section{Evaluación del proyecto}

Para la evaluación del proyecto de Innovación Docente INCLUREC y su evolución a lo largo del tiempo, definimos como objetivos:

- Analizar el número y tipo de recursos didácticos realizados a lo largo de la experiencia.

- Estudiar cuáles han sido las principales demandas en cuanto a los recursos se refiere, del alumnado con necesidades educativas especiales por parte del profesorado que los atiende.

- Realizar una descripción de los centros participantes.

- Profundizar sobre los resultados académicos del alumnado universitario.

\section{Participantes}

Los participantes en esta experiencia han sido 626 estudiantes universitarios que han elaborado los recursos didácticos y las fichas de los mismos. Profesorado de 78 centros educativos y 211 alumnos y alumnas con necesidades educativas. En el proceso de evaluación, hemos partido de las fichas de los universitarios, hemos contado con los testimonios de cuatro informantes clave (coordinadora del proyecto, una profesora de la universidad miembro del proyecto, una maestra de Educación Infantil y un estudiante de Magisterio) y con el feedback del profesorado en activo de los centros educativos.

Metodología de evaluación, instrumentos y análisis

La evaluación se está llevando a cabo a través de una metodología cualitativa utilizando como instrumentos las entrevistas, la observación y el análisis de documentos (fichas de los recursos, memorias de los proyectos educativos de los centros participantes y notas del alumnado universitario) En total se han analizado las 626 fichas de recursos, contrastándolo con las opiniones de los maestros/as implicados; 4 cuatro entrevistas y se ha hecho un seguimiento longitudinal de las notas del alumnado universitario.

Las fichas recogen información sobre: nombre del recurso, destinatario, áreas que se trabajan con el recurso, aspectos que desarrolla (contenidos), objetivos, actividades tipo, materiales, foto del recurso, paso a paso con fotos de la elaboración del recurso y observaciones u otros aspectos a aportar. Respecto a las entrevistas que se realizaron, tuvieron carácter semiestructurado con el objetivo de profundizar en aspectos relacionados con el inicio e implementación de la experiencia, la valoración de los participantes y las propuestas de mejora sugeridas para el futuro.

Para los análisis, el equipo de investigación elaboró un sistema de categorías a través de un proceso de codificación recurrente. A partir de dominios básicos, de forma inductiva, 
se han ido desarrollando las mismas. Llegamos a las siguientes categorías (se han seleccionado cuatro): características de los destinatarios, tipo de recursos elaborados, tipologías de los centros participantes y número y características del alumnado participante en el proyecto. Éstas nos han ayudado a realizar la descripción de los resultados contrastando la información obtenida a través de los instrumentos, elaborando así un DAFO que nos permite llegar a las conclusiones del estudio que se muestran más adelante.

\section{Resultados}

Durante diez cursos académicos, el alumnado de la Universidad de Huelva en las diferentes materias de diplomatura y ahora de Grado y Máster, han elaborado alrededor de 312 recursos educativos para centros, entidades y asociaciones de Huelva capital y provincia y alumnado concreto y otros 320 recursos de carácter voluntario no destinado en principio a ningún alumno o alumna determinado sino a disposición de centros, familias, asociaciones... que lo soliciten. Con ellos se ha tratado de responder a las necesidades y limitaciones de alumnos/as con necesidades educativas, y a los requerimientos de formación en competencias de los estudiantes universitarios desde una perspectiva de aprendizaje servicio.

Las características del alumnado destinatario que el alumno/a de la universidad ha tenido que valorar y observar previamente para la creación del recurso educativo han sido: motrices, cognitivas, de autonomía, de comunicación... Lógicamente la mayor parte de estas necesidades no podemos solventarlas, pero sí trabajarlas, estimularlas...de la forma más lúdica y motivadora posible, y ahí es donde se incide en el proceso de diseño de los materiales.

Es el propio profesorado de los centros educativos y/o asociaciones quienes seleccionan el alumnado al que se le va a realizar el recurso "quien trabaja con estos niños/as es quien pide los recursos...” (Entrevista a la coordinadora). La motivación de la selección suele estar relacionada con las características y necesidades del alumnado destinatario, si son alumnos de nuevo ingreso... En concreto, en estos años, se han elaborado recursos para 211 alumnos y alumnas (cuadro 1). Dentro de este número, casi la mitad (50,24\%) son alumnos y alumnas con algunas limitaciones de la movilidad (parálisis cerebral, distrofias, espinas bífidas...), pero este dato es comprensible si recordamos que en un principio este proyecto iba destinado exclusivamente a este tipo de alumnado. Otro grupo mayoritario es el de alumnos con alguna discapacidad cognitiva y alumnado con Trastorno de espectro autista TEA, como se puede observar en la siguiente tabla:

Para estos más de 200 alumnos y alumnas se han diseñado e implementado 312 recursos. Éstos han sido muy diversos y las demandas también han sido muy heterogéneas pues dependían de los destinatarios, de sus posibilidades e intereses, de su edad, así como de las dificultades que el profesorado tenía respecto a adaptaciones concretas que o bien eran recursos no disponibles en sus centros, o bien, tenían poca formación al respecto y desconocían cómo adaptarlos.

Las demandas principalmente han estado relacionadas con la estimulación sensorial $(27,56 \%)$ en el sentido de libros y mantas sensoriales, juguetes, móviles, etc.; juegos didácticos (18,91\%); o de lógica matemática $(14,74 \%)$ entre otros recursos, como podemos apreciar por cursos en el cuadro 2. 
Cuadro 1. Relación de recursos elaborados en INCLUREC en función de la tipología de la discapacidad del alumno/a al que va dirigido

\begin{tabular}{lc}
\hline \multicolumn{1}{c}{ TIPO DE DISCAPACIDAD } & PORCENTAJE DE RECURSOS ELABORADOS \\
\hline Motórica & $50,24 \%$ \\
Motórica-cognitiva & $12,80 \%$ \\
Cognitiva & $11,37 \%$ \\
TEA & $12,80 \%$ \\
TGD & $3,8 \%$ \\
Trastornos de lenguaje & $2,4 \%$ \\
TDAH & $1,9 \%$ \\
Sensorial-visual & $0,95 \%$ \\
Motórica-visual & $0,95 \%$ \\
Altas capacidades; auditiva; motórica-auditiva; & \\
$\quad$ cognitiva-visual; TEA-motórica; motórica- & Cada una de ellas en torno al $0,5 \%$ \\
lenguaje & \\
\hline
\end{tabular}

Fuente: Elaboración propia.

Las tipologías de los centros solicitantes son también diversas: Infantil, Primaria, Secundaria; capital, provincia; centros ordinarios, de especial o de compensatoria; asociaciones externas al ámbito educativo formal, etc. No hay una selección prevista a priori, ni una determinación inicial

Lo que me llamó la atención del proyecto es que lo importante son los niños, no son los
centros ni los maestros, los niños; por lo que se priorizan sus necesidades y ese es el
criterio de selección. (Maestra participante)

Generalmente, son los propios centros los que se ponen en contacto con nosotros para solicitar participar en el proyecto. Las fórmulas por las que conocen el proyecto son: haber participado en cursos y seminarios organizados en y por la Facultad; haber sido informados por el Equipo de Orientación Educativa de su zona o por el Centro de Profesores (CEP) de la Delegación de Educación con los que trabajamos y colaboramos; y en otras ocasiones, es el propio alumnado universitario quien les informa del proyecto y de sus posibilidades, o son ex alumnos/as que piden ayuda con un determinado caso:

Cuando llegué a mis prácticas en $3^{\circ}$ de Grado le dije a mi tutora que tenía que solicitar recursos a INCLUREC; considero que es una las experiencias más enriquecedoras que he vivido en la carrera y después en el máster. (Alumna participante)

Cuantificando los centros, en estos diez años el proyecto ha colaborado y ha desarrollado actividades de servicio en 78 centros entre los que podemos situar un $39,74 \%$ en la capital y un $60,26 \%$ en la provincia de Huelva. La mayoría son de Infantil y Primaria $(88,61 \%)$ y en menor medida IES (6,329\%) y Centros o Asociaciones de Educación Especial (1,2\%). Este último curso hemos comenzado a trabajar con Asociaciones relacionadas con la discapacidad, por lo que es previsible que aumenten los porcentajes referidos a este contexto:

Es una línea que hay que trabajar más porque no sólo se trabajan con recursos en los centros, sino que también en este tipo de asociaciones e incluso familias. (Coordinadora)

Este proyecto no hubiese sido posible sin la participación del alumnado de la Universidad que es quien en definitiva lleva el peso de este servicio: acuden a los centros, evalúan las necesidades del alumnado, realizan el recurso con la consiguiente necesidad de formación al respecto... 
Cuadro 2. Recursos elaborados en INCLUREC en función de la tipología del mismo y curso de elaboración

\begin{tabular}{|c|c|c|c|c|c|c|c|c|c|c|c|c|}
\hline & \multicolumn{10}{|c|}{ Curso } & \multirow[b]{2}{*}{$\mathbf{T}^{*}$} & \multirow[b]{2}{*}{$\%$} \\
\hline & $\begin{array}{c}08- \\
09\end{array}$ & $\begin{array}{c}09- \\
10\end{array}$ & $\begin{array}{c}10- \\
11\end{array}$ & $\begin{array}{c}11- \\
12\end{array}$ & $\begin{array}{c}12- \\
13\end{array}$ & $\begin{array}{c}13- \\
14 \\
\end{array}$ & $\begin{array}{l}14- \\
15\end{array}$ & $\begin{array}{c}15- \\
16\end{array}$ & $\begin{array}{c}16- \\
17\end{array}$ & $\begin{array}{c}17- \\
18\end{array}$ & & \\
\hline $\begin{array}{l}\text { Lógica- } \\
\text { matemática }\end{array}$ & 4 & 2 & 7 & 7 & 5 & 2 & 3 & 3 & 5 & 8 & 46 & 14,74 \\
\hline $\begin{array}{l}\text { Iniciación } \\
\text { lectoescrit. }\end{array}$ & 1 & 5 & 4 & 1 & 4 & 4 & 6 & 3 & 4 & 4 & 36 & 11,54 \\
\hline $\begin{array}{l}\text { Estimulación } \\
\text { sensorial }\end{array}$ & 6 & 11 & 8 & 10 & 12 & 9 & 3 & 7 & 11 & 9 & 86 & 27,56 \\
\hline Comunicación & 8 & 2 & 5 & 4 & $\mathrm{O}$ & 1 & $\mathrm{O}$ & $\mathrm{O}$ & 2 & 4 & 26 & 8,3 \\
\hline $\begin{array}{l}\text { Autonomía } \\
\text { personal y } \\
\text { social }\end{array}$ & 3 & 1 & 2 & 2 & 2 & 5 & 6 & 2 & 4 & 4 & 31 & 9,93 \\
\hline $\begin{array}{l}\text { Útiles de } \\
\text { lectura }\end{array}$ & 2 & 1 & 1 & 3 & o & $\mathrm{O}$ & 3 & $\mathrm{O}$ & O & $\mathrm{O}$ & 10 & 3,2 \\
\hline $\begin{array}{l}\text { Juegos } \\
\text { didácticos }\end{array}$ & 1 & O & 6 & 3 & 6 & 10 & 7 & 10 & 11 & 5 & 59 & 18,91 \\
\hline $\begin{array}{l}\text { Pulseras } \\
\text { lastradas }\end{array}$ & 2 & $\mathrm{O}$ & 2 & $\mathrm{O}$ & o & $\mathrm{O}$ & O & $\mathrm{O}$ & 1 & $\mathrm{O}$ & 5 & 1,6 \\
\hline Otros & 3 & 1 & 1 & $\mathrm{O}$ & 2 & 1 & 4 & 1 & 0 & $\mathrm{O}$ & 13 & 4,16 \\
\hline Totales & 30 & 23 & 36 & 30 & 31 & 32 & 32 & 26 & 38 & 34 & 312 & \\
\hline
\end{tabular}

Nota: * Total.

Fuente: Elaboración propia.

A lo largo de estos años, han sido muchos los alumnos y alumnas que se han formado respecto a la necesidad de dar una respuesta real al alumnado con necesidades educativas especiales que nos encontramos en los centros. Esta formación ha sido más o menos directa. Podemos cuantificar que en este periodo 1200 estudiantes del Grado de Educación Infantil y Primaria y del Máster Oficial en Educación Especial han conocido el proyecto, se han formado en él, han conocido los recursos elaborados y ofrecido a los centros. Entre estos, 626 han participado de forma directa en el proyecto realizando los recursos y con las actividades antes descritas, desde las diferentes materias de las antiguas diplomaturas y desde los actuales Grados y Master de Educación Especial de la Universidad de Huelva.

Un dato fundamental es que la participación en este proyecto no era obligatoria. Se define como una oportunidad de aprendizaje complementaria dentro del sistema reglado de materias. En los últimos cursos (desde el curso 14/15), sin embargo, en las asignaturas de máster se han incluido como tareas obligatorias, siendo valoradas positivamente por los estudiantes:

$$
\begin{aligned}
& \text { Yo siento que los estudiantes valoran mi asignatura gracias a INCLUREC, ellos dicen } \\
& \text { que elaborar una tarea que tiene un fin real les hace estar ilusionados; esto ha hecho } \\
& \text { que en los últimos cursos lo lleve a cabo de forma obligatoria. (Profesora } \\
& \text { participante) }
\end{aligned}
$$

Si nos referimos a los resultados académicos obtenidos por los alumnos y alumnas universitarios participantes en las asignaturas implicadas en el proyecto, se aprecian unos resultados muy favorables en términos cuantitativos. Así, por ejemplo, en la asignatura de grado Refuerzo pedagógico a la diversidad el $79,2 \%$ de los alumnos matriculados en los últimos cinco cursos han superado la asignatura. El porcentaje de alumnos de esa asignatura que han obtenido notas muy altas (Sobresalientes o Matrículas de Honor) 
durante esos cursos es del $21,9 \%$, siendo además positiva la evolución de este tipo de notas muy altas: han pasado de representar el 9,3\% en el curso 2013/14 al 38,3\% en curso 2017/18. Si atendemos a las asignaturas de máster que también incluían estas prácticas con carácter obligatorio (Discapacidad motórica y Alumnado con espectro autista) también se observan resultados académicos que indican un alto aprovechamiento de las enseñanzas impartidas (cuadro 3).

Cuadro 3. Resultados académicos del alumnado por curso académico y asignatura (en porcentaje)

\begin{tabular}{|c|c|c|c|c|c|}
\hline \multirow{2}{*}{ ASIGNATURA } & \multicolumn{5}{|c|}{ Curso } \\
\hline & $13-14$ & $14-15$ & $15-16$ & $16-17$ & $17-18$ \\
\hline $\begin{array}{l}\text { Refuerzo } \\
\text { pedagógico } \\
\text { a la } \\
\text { diversidad } \\
\text { del } \\
\text { alumnado } \\
\text { (Grado E. } \\
\text { Primaria) } \\
\end{array}$ & $\begin{array}{l}\text { MH: - } \\
\text { Sob: 9,23 } \\
\text { Not. 43,07 } \\
\text { Aprob: } 20,00 \\
\text { Susp: } 24,62 \\
\text { NP: } 3,08\end{array}$ & $\begin{array}{l}\text { MH: } 5,70 \\
\text { Sob: } 12,50 \\
\text { Not: } 45,90 \\
\text { Aprob: } 21,25 \\
\text { Susp: } 10,90 \\
\text { NP: } 3,75\end{array}$ & $\begin{array}{l}\text { MH: } 2,09 \\
\text { Sob: } 14,19 \\
\text { Not: } 40,79 \\
\text { Aprob: } 24,07 \\
\text { Susp: } 12,46 \\
\text { NP: } 7,71\end{array}$ & $\begin{array}{l}\text { MH: } 3,45 \\
\text { Sob: } 24,24 \\
\text { Not: } 38,64 \\
\text { Aprob: } \\
11,36 \\
\text { Susp: } 15,91 \\
\text { NP:6,40 }\end{array}$ & $\begin{array}{l}\text { MH: } 2,90 \\
\text { Sob: } 35,37 \\
\text { Not: } 39,73 \\
\text { Aprob: } 2,90 \\
\text { Susp: } 8,80 \\
\text { NP: } 10,30\end{array}$ \\
\hline $\begin{array}{c}\text { Discapacidad } \\
\text { Motórica } \\
\text { (Máster E. } \\
\text { Especial) }\end{array}$ & $\begin{array}{l}\text { MH: - } \\
\text { Sob: } 35,00 \\
\text { Not: } 45,00 \\
\text { Aprob: } 20,00 \\
\text { Susp: - } \\
\text { NP: - }\end{array}$ & $\begin{array}{l}\text { MH: - } \\
\text { Sobr: } 44,18 \\
\text { Not: } 34,88 \\
\text { Aprob: } 18,60 \\
\text { Susp: - } \\
\text { NP: 4,34 }\end{array}$ & $\begin{array}{l}\text { MH: - } \\
\text { Sobr: } 55,00 \\
\text { Not: } 28,50 \\
\text { Aprob: } 8,00 \\
\text { Susp: }- \\
\text { NP: } 8,50\end{array}$ & $\begin{array}{l}\text { MH: - } \\
\text { Sobr: } 48,78 \\
\text { Not: } 14,63 \\
\text { Aprob: } \\
29,26 \\
\text { Susp: } 2,46 \\
\text { NP: } 4,87 \\
\end{array}$ & $\begin{array}{l}\text { MH: } 2,59 \\
\text { Sob: } 53,24 \\
\text { Not: } 24,67 \\
\text { Aprob: } 16,88 \\
\text { Susp: - } \\
\text { NP: } 2,62\end{array}$ \\
\hline $\begin{array}{l}\text { Alumnado } \\
\text { con } \\
\text { Espectro } \\
\text { Autista } \\
\text { (Máster E. } \\
\text { Especial) }\end{array}$ & $\begin{array}{l}\text { MH: - } \\
\text { Sob: 42,50 } \\
\text { Not: } 55,00 \\
\text { Aprob: - } \\
\text { Susp: - } \\
\text { NP: 2,50 }\end{array}$ & $\begin{array}{l}\text { MH: - } \\
\text { Sob: } 60,47 \\
\text { Not: } 23,26 \\
\text { Aprob: } 6,98 \\
\text { Susp: - } \\
\text { NP: } 9,29\end{array}$ & $\begin{array}{l}\text { M. Honor - } \\
\text { Sob: } 35,29 \\
\text { Not: } 47.07 \\
\text { Aprob: } 5,88 \\
\text { Susp: - } \\
\text { NP: } 11,76\end{array}$ & $\begin{array}{l}\text { MH- } \\
\text { Sob: } 36,59 \\
\text { Not: } 43,90 \\
\text { Aprob: } \\
17.07 \\
\text { Susp: - } \\
\text { NP: } 2,44\end{array}$ & $\begin{array}{l}\text { MH: } 2,77 \\
\text { Sobr: } 62,53 \\
\text { Not: } 29,16 \\
\text { Aprob: } 1,38 \\
\text { Susp: - } \\
\text { NP: 4,16 }\end{array}$ \\
\hline
\end{tabular}

Fuente: Elaboración propia.

$\mathrm{Al}$ igual que en la asignatura de grado, tanto el porcentaje de alumnos que superan la asignatura (94,7\% de promedio de los últimos cinco cursos) como el porcentaje de notas muy altas (desde el 38,7\% en el 2013/14 al 60,6\% en el 2017/18) pueden ser considerados indicadores de muy buen rendimiento en esas asignaturas. Aportamos a modo de ejemplo algunos recursos elaborados por el proyecto en el presente curso (cuadro 4).

\section{Discusión y conclusiones}

Son diez cursos académicos los que llevamos trabajando con este modelo y las autoras de este trabajo hemos reflexionado sobre los beneficios y necesidades-dificultades que encontramos en este tipo de experiencias docentes. Nuestro proyecto no trata de una intervención institucionalizada oficialmente. Por ejemplo, en experiencias como la de Freixa y otros (2013) de la Universidad de Barcelona han creado una oficina dependiente del decanato para dinamizar, liderar y hacer un seguimiento de las experiencias de ApS. Esto mismo sucede con el de la Universidad de Zaragoza (Arranz, Vázquez y Abán, 2013). En el proyecto de la UHU, el establecimiento de redes de colaboración con la firma de 
convenios, el apoyo al alumnado y la evaluación de la propuesta, se hace desde la coordinación y con el grupo de profesoras implicadas en diferentes materias (dos o tres anualmente de manera estable). Ello nos lleva a plantear líneas futuras de trabajo que den soporte y logren ampliar y dar más visibilidad al proyecto.

Cuadro 4. Algunos recursos elaborados en INCLUREC durante el curso 2017-18 con referencia al alumnado destinatario

\begin{tabular}{ll}
\hline \multicolumn{1}{c}{ RECURSO ELABORADO } & \multicolumn{1}{c}{ ALUMNADO DESTINATARIO } \\
\hline $\begin{array}{c}\text { Muñeco partes del cuerpo; Paneles } \\
\text { partes de la cara; Ruleta de emociones }\end{array}$ & $\begin{array}{c}\text { Aula específica. } 4 \text { alumnos 14, 11,12, 10 años con } \\
\text { discapacidad cognitiva }\end{array}$ \\
\hline El tocador de Blanca (comunicación) & 18 años TEA. Discapacidad cognitiva \\
\hline Sistema de comunicación & Alumna 5 años TGD \\
\hline $\begin{array}{l}\text { Emparejamiento de imágenes; Recursos } \\
\text { método Teacch }\end{array}$ & Alumno 9 años. Retraso, TEL, rasgos autistas \\
\hline Botellas sensoriales; Pisadas sensoriales & $\begin{array}{c}\text { Aula específica, alumno discapacidad motora; 10 } \\
\text { años }\end{array}$ \\
\hline Máquina de sumar; Máquina de restar & Alumno 8 años $\left(2^{\circ}\right.$ Primaria) con S. Asperger \\
\hline $\begin{array}{l}\text { Abaco; Fichas 1-100; Libro de } \\
\text { emociones }\end{array}$ & Aula especifica alumnado TEA \\
\hline Tablero interactivo; Láminas imantadas & 4 años Síndrome x Frágil. 6 años Parálisis cerebral \\
\hline Pizarra imantada matemáticas (futbol) & Parálisis cerebral 7 años en $2^{\circ}$ \\
\hline 6 bandejas de luz para diversos centros & Diversos centros y aulas \\
\hline Ruleta silábica; Libro sensorial & Clase de $2^{\circ}$ con un niño TADH y una niña TDA \\
\hline Libro sensorial & Niño 9 años con retraso grave \\
\hline El juego de las sílabas; Panel sensorial & 6 años síndrome Prader-Willi \\
\hline Alfombra sensorial; Caja sensorial & Niño 3 años TEA \\
\hline El escenario del 10o (ABN) & Alumno con 14 años TEA \\
\hline Fuente: Elaboración propia. &
\end{tabular}

El proyecto tal y como hemos ido describiendo tiene dificultades y fortalezas. Comenzando por las primeras, podemos referirlas a los dos vértices del proyecto: universidad y centros. Respecto al primer contexto, es una limitación la dificultad de llevarlo a cabo con los recursos con los que contamos. El peso del trabajo recae en el profesorado universitario, mientras que la evaluación del alumnado para el que se diseña el material, es realizado por los estudiantes universitarios en colaboración con maestros, tutores, maestros/as de apoyo,...Exige una formación del alumnado, tiempos de tutorías, coordinación de los grupos... y este proceso no siempre se entiende como una formación para atender a la diversidad; no siempre es contemplada como un desarrollo de competencias imprescindible para desarrollar una opción profesional futura. A veces se percibe como una práctica "más o menos interesante" por parte de los propios docentes. También apreciamos como limitación la poca visibilización del proyecto dentro de la Facultad por parte del profesorado y alumnado de otras menciones que no sean las de Educación Especial. Por otro lado, desde los centros, en estos años hemos encontrado limitaciones en el momento de ofrecer información de las características de determinados alumnos y alumnas, la imprecisión de las demandas y en otros la falta de contextualización a la hora de solicitar "imposibles” al alumnado del Grado o Máster.

Como fortalezas, los niveles de participación tanto del alumnado universitario como del profesorado de los centros solicitantes de recursos son muy altos. Junto a ello, los resultados académicos de los estudiantes en formación son positivos y en las evaluaciones 
de la asignatura muestran una alta satisfacción año tras año. Ferguson, Hanreddy y Draxton (2011) afirman que, si escuchamos a nuestros estudiantes, lograremos mejorar su compromiso en los procesos de toma de decisiones sobre los entornos -y nosotros añadimos- procesos de aprendizaje. Contribuiremos a mejorar su motivación e involucración. En nuestro caso, implicar a los estudiantes también significa adoptar un posicionamiento ideológico respecto al campo de investigación. Como afirma Messiou (2008, basándose en varios estudios; Messiou, 2014), desde esta postura estamos considerando al alumnado como iguales, como aliados y como herramientas clave para la mejora de las escuelas, la enseñanza y los aprendizajes. Como indican en este sentido Muntaner, Roselló y De la Iglesia, (2016) los logros de todo el alumnado y estos avances significan cambios importantes en el sistema educativo y en el desarrollo de escuelas inclusivas.

Por todo ello y para un mejor desarrollo de estos programas y experiencias, creemos que en el futuro sí debemos reforzar algunas cuestiones:

- Se debería realizar una ampliación de créditos ECTS tanto para los alumnos/as como en el plan de organización docente para el profesorado. Un mayor tiempo para la materia ayudaría a desarrollar más las clases teniendo tiempo con el alumnado, y a éstos a disponer de más espacios para el aprendizaje.

- Creemos que el proyecto enriquece el plan de estudios y podría extenderse a otras materias si se logra tener un respaldo institucional.

- Es necesario visibilizar y difundir aún más el proyecto en la facultad y centros educativos, así como en las asociaciones.

Aplicando un modelo empresarial de evaluación DAFO (Debilidades, Amenazas, Fortalezas y Oportunidades;), que ha sido empleado en otras experiencias de ApS como la de Arranz, Vázquez y Abán (2013), podemos resumir la evaluación de nuestra propuesta (cuadro 5).

La reflexión sobre el método de trabajo que seguimos en el ApS nos lleva a considerar la existencia de algunas asociaciones, señaladas por Monroy y Hernández Pina (2014), como la "asociación entre el enfoque profundo de aprendizaje y la percepción de libertad para aprender” (p. 115), “y entre las formas de enseñar... caracterizada por apoyo al estudiante, feedback, libertad para aprender, objetivos claros y con relevancia para la práctica profesional" (p. 114), que en nuestra experiencia tratamos de garantizar. Para ello, creemos en el futuro el proyecto debería incluir herramientas de evaluación de los rendimientos específicas y desarrolladas a partir de algunos proyectos europeos. Además, debemos mejorar el sistema de seguimiento del proyecto, no sólo por la posible ampliación a nuevos colegios, institutos o asociaciones, sino por mejorar los aprendizajes y adquisición de competencias de nuestro alumnado. Así por ejemplo creemos interesante utilizar medidas de "aprendizaje para aprender" de Fredriksson y Hoskins (2008); cómo trabajar y definir los resultados de aprendizaje (Cedefop, 2011); así como otras herramientas definidas dentro del trabajo de la Unión Europea centrado en la evaluación de competencias (Comisión Europea, 2012). La ejecución de este enfoque de ApS es compleja y necesita de mayor respaldo institucional y una mayor difusión, tal y como adelantábamos. Paralelamente, las colaboraciones con los centros se están organizando mediante convenios. Y esperamos mejorar la evaluación del proyecto y del diseño y uso de los recursos educativos, gracias a la retroalimentación que recibiremos a través de un cuestionario elaborado ad hoc. Éste ha sido validado recientemente y va dirigido al 
profesorado en activo y a nuestro alumnado para realizar la evaluación y seguimiento del uso del recurso, así como los avances educativos y de integración del alumno/a para el que se ha diseñado. Estamos de acuerdo con Fiuza y Sierra (2017) y Gezuraga (2017) en que el desarrollo de una experiencia de ApS supone una importante aportación a nuevos modos de encarar el proceso de enseñanza-aprendizaje en la universidad, generando cambios en la forma en que se enseña a los futuros maestros y en cómo éstos aprenden, pero también en el estilo docente que en el futuro desarrollarán en sus aulas.

Cuadro 5. Evaluación del proyecto INCLUREC empleando modelo DAFO

Profesorado universitario implicado en las materias y altamente motivado

Buen clima y colaboración entre profesorado (de distintos niveles) y profesionales de asociaciones

Fortalezas

Se conecta el trabajo entre materias tanto de grado como de posgrado

Alta motivación del alumnado y alta participación

Buenos rendimientos en la evaluación del alumnado universitario

Satisfacción en las evaluaciones de las asignaturas participantes en el proyecto, por parte del alumnado universitario

Poca implicación de otro profesorado ajeno al proyecto

No se difunde a nivel interno

Supone una mayor inversión de tiempo y trabajo previo por parte del profesorado universitario

No hay reconocimiento institucional

Debilidades Dificultades temporales para planificar y coordinar la experiencia por parte del profesorado implicado

Sobrecarga de trabajos en el caso del alumnado universitario

Debemos mejorar los sistemas de evaluación de los recursos haciendo un mayor seguimiento de su uso en el aula

Debemos mejorar los instrumentos de evaluación de las competencias que el alumnado universitario adquiere

El profesorado de enseñanzas medias participa activamente y demanda la colaboración activamente

El proyecto se difunde por el "boca a boca” y cada vez hay más demandas por parte de los centros educativos

La satisfacción por los recursos elaborados y el trabajo que el

Oportunidades alumnado universitario realiza, por parte del profesorado de los centros educativos es muy alta

Los EOEs de la zona colaboran activamente en darle una mayor visibilidad al proyecto formativo de ApS

Las familias están empezando a participar y a demandar adaptaciones para mejorar las condiciones y recursos en el hogar

No se trabaja a nivel institucional universitario

Los profesores jóvenes no tienen estabilidad laboral y son los que más suelen participar en estas experiencias

Amenazas Aunque los EOEs, y Delegación difunden el proyecto, no recibimos un apoyo institucional para mejorar la colaboración entre centros educativos-universidad, ni en la elaboración de los recursos.

Cada vez es más complejo el acceso a los centros educativos

Fuente: Elaboración propia. 
Para nosotros es un reto personal y profesional mejorar la formación de los y las futuros docentes y una experiencia enriquecedora profesional y personalmente sobre la que deseamos seguir avanzando. Esta experiencia de ApS es un intento más de garantizar oportunidades de aprendizaje para todo el alumnado, sin olvidar la complejidad del cómo lograrlo (Kyburiene y Navickiene, 2015; Romero-Jeldres et al., 2018)

\section{Agradecimientos}

Experiencia financiada por las ayudas a Proyectos de Innovación Docente del Plan Propio de la Universidad de Huelva de todos los cursos desde el 2007.

\section{Referencias}

Aramburuzabala, P. (2013). Aprendizaje-servicio: Una herramienta para educar desde y para la justicia social. Revista Internacional de Educación para la Justicia Social, 2(2), 5-1 1.

Arranz, P., Vázquez, S. y Abán, A. (2013). El proyecto de aprendizaje servicio en la Universidad de Zaragoza: Un análisis DAFO. En L. Rubio, E. Prats y L. Gómez (Coord.), Universidad y sociedad. Experiencias de aprendizaje servicio en la universidad (pp. 299-304). Barcelona: Universidad de Barcelona.

Bigg, J. (2003). Teaching for quality learning at university. Buckinghamshire: Society for Research into Higher Education and Open University Press

Cedefop. (2011). Using learning outcomes. Recuperado de: http://ec.europa.eu/education/lifelonglearning-policy/doc/eqf/note4_en.pdf

Comisión de las Comunidades Europeas. (2005). Recomendaciones del parlamento europeo y del consejo de Europa sobre las competencias clave para el aprendizaje permanente. Recuperado de http://eurlex.europa.eu/LexUriServ/site/es/com/2005/com2005_0548es01.pdf

Comisión Europea. (2012). Education and training 2020 work programme thematic working group 'assessment of key competences' literature review, glossary and examples. Recuperado de http://ec.europa.eu/dgs/education_culture

Consejo de la Unión Europea. (2009). Conclusiones del consejo de 12 de mayo de 2009 sobre un marco estratégico para la cooperación europea en el ámbito de la educación y la formación ("ET 2020") Diario Oficial $n^{\circ}$ C 119 de 28/05/2009. Recuperado de https://eur-lex.europa.eu/legalcontent/ES/TXT/?uri=CELEX:52009XG0528(01)

Ferguson, D., Hanreddy, A. y Draxton, S. (2011). Giving students voice as a strategy for improving teacher practice. London Review of Education, 9(1), 55-70.

https://doi.org/10.1080/14748460.2011.550435

Fernández March, A. (2006). Metodologías activas para la formación de competencias. Educatio Siglo XXI, 24, 35-56.

Fredriksson, U. y Hoskins, B. (2008). Learning to learn: What is it and can it be measured? Ispra: European Commission.

Freixa, M., Puig, J. M., Martín, X. y Escofet, A. (2013). Hacia la institucionalización del ApS en la universidad. En L. Rubio, E. Prats y L. Gómez (Coords.), Universidad y sociedad. Experiencias de aprendizaje servicio en la universidad (pp. 312-315). Barcelona: Universidad de Barcelona.

Häkkinen, P., Järvelä, S., Mäkitalo-Siegl, K., Ahonen, A., Näykki, P. y Valtonen, T. (2017). Preparing teacher-students for twenty-first-century learning practices: A framework for 
enhancing collaborative problem-solving and strategic learning skills. Teachers and Teaching, 23(1), 25-41. https://doi.org/10.1080/13540602.2016.1203772

Gargallo López, B., Jiménez Rodríguez, M. A., Martínez Hervás, N., Giménez Beut, J. A. y Pérez Pérez, C. (2017). Métodos centrados en el aprendizaje, implicación del alumno y percepción del contexto de aprendizaje en estudiantes universitarios. Educación XX1, 20(2), 161-187. https://doi.org/10.5944/educxx 1.19036

Gezuraga, M. (2017). El aprendizaje-servicio y su contribución a la función de extensión universitaria. Desarrollo en la Universidad del País Vasco (UPV/EHU). REICE. Revista Iberoamericana sobre Calidad, Eficacia y Cambio en Educación, 15(1), 5-18. https://doi.org/10.15366/reice2017.15.1.001

Fiuza, M. J. y Sierra, S. (2017). Creando y adaptando materiales para compartir: Una experiencia con futuros maestros. Educatio Siglo XXI, 35(3), 153-174. https://doi.org/10.6018/j/308951

García, M. y Cotrina, M. (2015). Aprendizaje y servicio (ApS) en la formación del profesorado: Haciendo efectiva la responsabilidad social y el compromiso ético. Profesorado. Revista de Currículum y Formación, 19(1), 1-3.

Gil-Gómez, J., Moliner-García, O., Chiva-Bartoll, O. y García López, R. (2016). Una experiencia de aprendizaje-servicio en futuros docentes: Desarrollo de la competencia social y ciudadana. Revista Complutense de Educación, 27(1), 53-73.

https://doi.org/10.5209/rev_RCED.2016.v27.n1.45071

Kyburiene, L. y Navickiene, G. (2015). The value of the model of a socially integral teaching/learning environment in the classroom from the point of view of learners who tend to socially withdraw. Citizenship, Social and Economic Education, 14(2), 133-147. https://doi.org/10.1177/2047173415603000

Kugelmass, J. W. (2001). Collaboration and compromise in creating and sustaining an inclusive school. International Journal of Inclusive Education, 5(1), 47-65. https://doi.org/10.1080/13603110121498

Mayor Paredes, D. y Rodríguez Mar, D. (2015). Aprendizaje-servicio: Construyendo espacios de intersección entre la escuela-comunidad-universidad. Profesorado. Revista de Currículum y Formación del Profesorado 19(1), 262-279.

Messiou, K. (2008). Understanding children's construction of meanings about other children: Implications for inclusive education. Journal of Research in Special Educational Needs, 8(1), 27-36. https://doi.org/10.1111/j.1471-3802.2008.00099.x

Messiou, K. (2014). Responding to diversity by engaging with students' voices. A strategy for teacher development. Accounts of practice. Southampton: European Union.

Monroy, F. y Hernández Pina, F. (2014). Factores que influyen en los enfoques de aprendizaje universitario. Una revisión sistemática. Educación XX1, 17(2), 105-124. https://doi.org/10.5944/educxx 1.17.2.11481

Montes, R., Tapia, M. y Yaber, L. (2011). Manual para docentes y estudiantes solidarios. Buenos Aires: CLAYSS.

Muntaner, J. J. Rosselló, M. R. y De la Iglesia, B. (2016). Buenas prácticas en educación inclusiva. Educatio Siglo XXI, 34(1), 31-50. https://doi.org/10.6018/j/252521

Murillo, F. J. (2012). Escuelas de calidad para la transformación social. REICE. Revista Iberoamericana sobre Calidad, Eficacia y Cambio en Educación, 10(1), 3-4.

Murillo, F. J. y Hernández-Castilla, R. (2014). Liderando escuelas justas para la justicia social. Revista Internacional de Educación para la Justicia Social, 3(2), 11-30. 
Murillo, F. J., Martínez-Garrido, C. y Belavi, G. (2017). Sugerencias para escribir un buen artículo científico en educación. REICE. Revista Iberoamericana sobre Calidad, Eficacia y Cambio en Educación, 15(3), 5-34. https://doi.org/10.15366/reice2017.15.3.001

Páez, M. y Puig, J. M. (2013). La reflexión en el aprendizaje-servicio. Revista Internacional de Educación para la Justicia Social, 2(2), 13-22.

Ponsa, P., Amante, B., Roman, J. A., Oliver, S., Díaz, M. y Vives, J. (2009). Higher education challenges: Introduction of active methodologies in engineering curricula. International Journal of Engineering Education, 25(4), 799-813.

Puig, J. M. y Palos, J. (2006). Rasgos pedagógicos del aprendizaje-servicio. Cuadernos de Pedagogía., 357, 60-63.

Puig, J. M. (2009). Aprendizaje servicio. Educación y compromiso cívico. Barcelona: Graó.

Puig, J. M., Gijón, M., Martín, X. y Rubio, L. (2011). Aprendizaje-servicio y educación para la ciudadanía. Revista de Educación, 298, 45-67.

Puig, J. M., Batlle, R., Bosch, C., Cerda, M., Climent, T., Gijón, M., ... y Trilla, J. (2009). Aprendizaje servicio. Educación y compromiso. Barcelona: Graó.

Rodríguez Herrero, P., De la Herrán, A. y Cortina Selva, M. (2015). Pedagogía de la muerte mediante aprendizaje de servicio. Educación XX1, 18(1), 189-212.

https://doi.org/10.5944/educxx 1.18.1.12317

Romero-Jeldres, M., Ramos, R., Castillo, C., Pérez, D. y Hernández, N. (2018). Aprendizaje servicio en la educación superior: Desde la participación individual a la participación institucionalizada. RIDAS. Revista Iberoamericana de Aprendizaje Servicio, 5, 123-141.

Root, S. (2005). Improving service-learning practice: Research on models to enhance impact. En S. Root, J. Callahan y S. Billig (Eds.), The national service-learning in teacher education partnership: A research retrospective. Greenwich: Information Age.

Rué, J. (2006). Disfrutar o sufrir la escolaridad obligatoria. ¿Quién es quién ante las oportunidades escolares? Barcelona: Octaedro.

Sánchez Moreno, M. (2007). Cómo enseñar en las aulas a través del estudio de casos. Zaragoza: ICE.

Tapia, M. N. (2008). Aprendizaje y servicio solidario. Buenos Aires: Ciudad Nueva.

Ubieto, I., Domingo, M., García, F. J. y Paulo, A. (2008). Practicar con la teoría: Metodologías activas en información y documentación en el marco del Espacio Europeo de Educación Superior. Zaragoza: Prensas Universitarias de Zaragoza.

Unión Europea. (2007). Conclusiones del consejo de la unión europea (y de los representantes de los gobiernos de los Estados miembros, reunidos en el seno del Consejo), sobre la mejora de la calidad de la educación del profesorado. Recuperado de https://es.slideshare.net/acortesalegre/conclusiones-del-consejo-de-europa-sobre-lamejora-de-la-calidad-del-profesorado-2007

Zabalza, M. A. y Zabalza, M. A. (2010). Planificación de la docencia en la universidad: Elaboración de las guías docentes de las materias. Madrid: Narcea Ediciones.

\section{Breve CV de las autoras}

\section{Inmaculada Gómez-Hurtado}

Doctora en Psicopedagogía y Profesora en el área de Didáctica de las Ciencias Sociales de la Facultad de Educación, Psicología y Ciencias del Deporte de la Universidad de Huelva. 
Sus principales líneas de investigación se desarrollan en el campo de la atención a la diversidad y la inclusión educativa, el liderazgo educativo y el estudio de la aspectos organizativos y didácticos en los de centros educativos. Información del grupo de investigación al que pertenece: http://www.uhu.es/doce. Pertenece a la Red RILME: http://www.rilme.org/. ORCID ID: https://orcid.org/0000-0002-0843-5784. Email: inmaculada.gomez@ddcc.uhu.es

\section{Asunción Moya Maya}

Profesora Titular del Departamento de Pedagogía de la Universidad de Huelva. Miembro del Grupo de Investigación DOCE de la citada universidad. Su investigación y docencia se relaciona principalmente con temáticas relativas a la atención a la diversidad, inclusión y apoyo educativo al alumnado con necesidades educativas especiales, de las que ha dirigido numerosas tesis doctorales y publicado diversos artículos y libros. Información del grupo de investigación al que pertenece: http://www.uhu.es/doce. ORCID ID: https://orcid.org/o000-0002-8039-7719. Email: asuncion@dedu.uhu.es

\section{Pilar García-Rodríguez}

Profesora Titular del Departamento de Pedagogía de la Universidad de Huelva y actualmente su directora. Investigadora Principal del Grupo de Investigación DOCE (http://www.uhu.es/doce) y miembro activo de RILME (Red de Investigación sobre Liderazgo y Mejora de la Escuela, http://www.rilme.org/.). Su investigación y docencia se relaciona principalmente con temáticas relativas a la evaluación de la mejora de la escuela, desarrollo del liderazgo y atención a la diversidad. ORCID ID: http://orcid.org/o000-0003-4796-781X.Email:mpgarcia@uhu.es 